\title{
Dispersal and metapopulation stability
}

Shaopeng Wang, Bart Haegeman, Michel Loreau

Metapopulation dynamics are jointly regulated by local and spatial factors. These factors may affect the dynamics of local populations and of the entire metapopulation differently. Previous studies have shown that dispersal can stabilize local populations; however, as dispersal also tends to increase spatial synchrony, its net effect on metapopulation stability has been controversial. Here we present a simple metapopulation model to study how dispersal, in interaction with other spatial and local processes, affects the temporal variability of metapopulations in a stochastic environment. Our results show that in homogeneous metapopulations, the local stabilizing and spatial synchronizing effects of dispersal cancel each other out, such that dispersal has no effect on metapopulation variability. This result is robust to moderate heterogeneities in local and spatial parameters. When local and spatial dynamics exhibit high heterogeneities, however, dispersal can either stabilize or destabilize metapopulation dynamics through various mechanisms. Our findings have important theoretical and practical implications. We show that dispersal functions as a form of spatial intraspecific mutualism in metapopulation dynamics and that its effect on metapopulation stability is opposite to that of interspecific competition on local community stability. Our results also suggest that conservation corridors should be designed with appreciation of spatial heterogeneities in population dynamics in order to maximize metapopulation stability. 


\section{Dispersal and metapopulation stability}

2

3 Shaopeng Wang, Bart Haegeman and Michel Loreau

4 Centre for Biodiversity Theory and Modelling, Station d'Ecologie Experimentale du CNRS,

5 09200, Moulis, France

6 Email: shaopeng.wang@ecoex-moulis.cnrs.fr, bart.haegeman@ecoex-moulis.cnrs.fr,

7 michel.loreau@ecoex-moulis.cnrs.fr

10 Keywords:

11 metapopulation, stability, variability, dispersal, synchrony, spatial heterogeneity, asymmetry, 12 corridor.

\section{Author for correspondence:}

16 Shaopeng Wang

17 Centre for Biodiversity Theory and Modelling, Station d'Ecologie Expérimentale du CNRS,

1809200 Moulis, France. Tel: +33-561040360, Fax: +33-561960851, Email:

19 shaopeng.wang@ecoex-moulis.cnrs.fr 


\section{Abstract}

Metapopulation dynamics are jointly regulated by local and spatial factors. These factors may affect the dynamics of local populations and of the entire metapopulation differently. Previous studies have shown that dispersal can stabilize local populations; however, as dispersal also tends to increase spatial synchrony, its net effect on metapopulation stability has been controversial. Here we present a simple metapopulation model to study how dispersal, in interaction with other spatial and local processes, affects the temporal variability of metapopulations in a stochastic environment. Our results show that in homogeneous metapopulations, the local stabilizing and spatial synchronizing effects of dispersal cancel each other out, such that dispersal has no effect on metapopulation variability. This result is robust to moderate heterogeneities in local and spatial parameters. When local and spatial dynamics exhibit high heterogeneities, however, dispersal can either stabilize or destabilize metapopulation dynamics through various mechanisms. Our findings have important theoretical and practical implications. We show that dispersal functions as a form of spatial intraspecific mutualism in metapopulation dynamics and that its effect on metapopulation stability is opposite to that of interspecific competition on local community stability. Our results also suggest that conservation corridors should be designed with appreciation of spatial heterogeneities in population dynamics in order to maximize metapopulation stability. 
Introduction

41 One important question in spatial ecology is how spatial coupling of local populations affects the dynamics and stability of metapopulations. Natural populations undergo various sources of stochasticity and fluctuate permanently over time (Lundberg et al. 2000). A common empirical measure of stability is the temporal variability of population size (Pimm 1984, Ives 1995), which is closely related to the long-term persistence of the population (Inchausti and Halley 2003). Within a metapopulation, temporal variability can be measured at both the local population and metapopulation scales. Ecological processes, particularly dispersal, may affect the variability at different scales differently (Dey and Joshi 2006, Vogwill et al. 2009).

At the local scale, theoretical and empirical studies have widely documented that dispersal can decrease population variability and hence local extinction rate through spatial averaging or rescue effects (Lande et al. 1998, Briggs and Hoopes 2004, Vogwill et al. 2009, Abbott 2011). However, the effect of dispersal on metapopulation stability, i.e. the stability of the whole metapopulation, has been controversial. While stabilizing local dynamics, dispersal also tends to increase the spatial synchrony between local populations (Kendall et al. 2000, Ripa 2000, Liebhold et al. 2004, Abbott 2011). Such synchronizing effects have been demonstrated to impair the persistence and stability of metapopulations (Heino et al. 1997, Earn et al. 2000, Gouhier et al. 2010; but see Blasius et al. 1999). Therefore, dispersal is a double-edged sword (Hudson and Cattadori 1999): it can either decrease metapopulation variability and extinction rate through its local stabilizing effects or increase them through its spatial synchronizing effects. Experimental studies have reported stabilizing, destabilizing, or no effects of dispersal on metapopulation variability (Dey and Joshi 2006, Vogwill et al. 2009, Steiner et al. 2013). Given these controversies, it is necessary to use theoretical models to quantitatively study the two 
63 effects of dispersal together and understand the net effects of dispersal on stability at the

64 metapopulation scale.

65 Recently, we have developed a general framework that links population or ecosystem 66 stability across multiple scales (Wang and Loreau 2014). When applied to a single-species metapopulation, this framework shows that metapopulation variability can be calculated as the product of local population variability and a metapopulation-wide measure of spatial synchrony. Consequently, the net effect of dispersal on metapopulation variability is determined by the relative strengths of its local stabilizing and spatial synchronizing effects. This framework variability scale up from population to metapopulation scales.

In this study, we use simple metapopulation models to investigate analytically how dispersal, in interaction with other factors, regulates temporal variability at different scales. In all our models, local populations have feasible and stable equilibria in the absence of environmental stochasticity; but due to environmental stochasticity, populations fluctuate permanently around these equilibria. This assumption allows us to analyze our models with the linearization approximation (see Methods). We first study a homogeneous metapopulation in which local (i.e. local intrinsic growth rate and carrying capacity) and spatial (i.e. dispersal) parameters are all identical among patches. In this case, we derive analytic formulae that quantify the local stabilizing and spatial synchronizing effects of dispersal. Interestingly, we show that these two effects cancel each other out, such that dispersal has no net effect on metapopulation variability. We then consider more general cases with spatially heterogeneous population dynamics and/or asymmetric dispersal rates. Spatial heterogeneities in the environment (e.g. temperature, patch size, etc.) can result in variation in population dynamics among patches (Brown et al. 2004, 
Strevens and Bonsall 2011; De Roissart et al. 2015). In addition, dispersal can exhibit directionality due to abiotic (e.g. water or wind flows; see Levine 2003, Anderson et al. 2012) or biotic (e.g. active dispersal; see Pulliam 1988, Bowler and Benton 2005) factors. It remains unexplored how spatial heterogeneities in population dynamics and asymmetric dispersal interact and affect the stability of metapopulations at multiple scales (but see Dey et al. 2014). By studying metapopulation models with heterogeneous local and spatial parameters, we identify several mechanisms by which dispersal can increase or decrease metapopulation variability. Finally, we discuss the implications of our model for landscape management.

\section{Methods}

\section{Model}

Consider a metapopulation composed of $m$ local patches. The dynamics of local populations are governed by density-dependent growth and density-independent dispersal between patches. We use a continuous-time model as follows:

$\frac{d N_{i}(t)}{d t}=r_{i} N_{i}(t) \cdot\left[1-\frac{N_{i}(t)}{k_{i}}\right]-d_{i} N_{i}(t)+\sum_{j \neq i} \frac{d_{j}}{m-1} \cdot N_{j}(t)+N_{i}(t) \varepsilon_{i}(t)$

where $N_{i}(t)$ represents the population size (or biomass) in patch $i$ at time $t, r_{i}$ and $k_{i}$ represent the intrinsic growth rate and carrying capacity in patch $i$, respectively, and $d_{i}$ represents the rate for each individual in patch $i$ to immigrate into other patches. Here, we consider an implicit spatial structure so that individuals from patch $i$ have equal probabilities to reach any other patch $\left(d_{i} /(m-\right.$ 1)). The random variables $\varepsilon_{\mathrm{i}}(t)$ represent environmental stochasticity in the growth rate of population $i$ at time $t$. For simplicity, we assume that the environmental stochasticity is 
108 independent through time (i.e. white noise). The spatial correlation of the white-noise variables

$109 \varepsilon_{\mathrm{i}}(t)$ are characterized by the covariance matrix $V_{\varepsilon}$, for which we assume the following symmetric

110 structure: $V_{\varepsilon}(i, i)=\sigma^{2}$ for any $i$ and $V_{\varepsilon}(i, j)=\rho \sigma^{2}$ for any $i \neq j$ (see Appendix 1 for details).

111 Particularly, $\rho$ represents the between-patch correlation in population environmental responses.

112 Previous studies have often used discrete-time models to study metapopulation dynamics

113 in stochastic environments (e.g. Kendall et al. 2000, Ripa 2000, Abbott 2011). In these models,

114 the order of spatial and local processes can alter the results quantitatively (Ripa 2000). Our

115 continuous-time model avoids this problem. In order to compare it with previous models,

116 however, we also study two discrete-time models that capture essentially the same spatial and

117 local processes, with distinct orderings of these processes (Appendix 3). The results under the

118 discrete-time models are qualitatively similar to those of the continuous-time model (except for

119 the effects of the intrinsic growth rate $r$; see Appendix 3). We thus present only the continuous-

120 time model in the main text. Interested readers can find all the details about the discrete-time

121 models in Appendix 3.

122

\section{Solving for the covariance matrix}

124 A common approach to study the temporal variability of stochastic dynamical systems is to

125 linearize the system around its stable equilibrium (Ives 1995, Lundberg et al. 2000, Ripa and Ives

126 2003, Greenman and Benton 2005). This linearization approach provides approximate analytic

127 solutions for the stationary covariance matrix, on condition that the dynamical system has a

128 stable equilibrium in the absence of stochasticity and undergoes moderate stochasticity. With this

129 approach, we first derive the analytic solutions for homogeneous metapopulations in which local

130 and spatial dynamics have identical parameters, i.e. for any $i, r_{i}=r, k_{i}=k, d_{i}=d$. In this case, the 
131 equilibrium local population size is simply $N^{*}=k$. Around $N_{i}(t)=N^{*}$ and $\varepsilon_{i}(t)=0$, Eq. 1 can be

132 linearized into the following form (see Appendix 1):

$133 \quad \frac{d \vec{X}(t)}{d t}=J \vec{X}(t)+N^{*} \vec{\varepsilon}(t)$

134 where:

$$
\begin{aligned}
& \vec{X}(t)=\left(N_{1}(t)-N^{*}, \quad \square, \quad N_{m}(t)-N^{*}\right)^{\prime} \\
& \stackrel{\square}{\varepsilon}(t)=\left(\varepsilon_{1}(t), \quad \square, \quad \varepsilon_{m}(t)\right)^{\prime}
\end{aligned}
$$

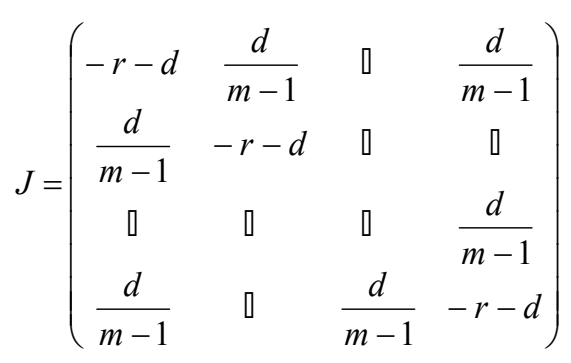

138 Note that $J$ is the Jacobian matrix, and the equilibrium is locally stable when $r>0$ (see Appendix

139 1). Under the assumption of white noise, we can derive from Eq. 2 the stationary covariance

140 matrix of metapopulation dynamics $\left(V_{N}=\operatorname{Cov}(\vec{X}(\infty))\right)$, which is the solution of the following

141 equation (van Kampen, 1992; see Appendix 1):

$142 V_{N} J+J V_{N}+N^{* 2} \cdot V_{\varepsilon}=0$

143 For general cases with heterogeneous local and spatial parameters, we analyze two-patch

144 metapopulation models using similar procedures as above (see Appendix 2). First, by ignoring environmental fluctuations, we compute the equilibrium local population sizes $\left(N_{1}{ }^{*}, N_{2}{ }^{*}\right)$ numerically. Our simulations suggest that there is always one globally stable equilibrium, i.e.

147 trajectories starting from different initial conditions all converge to the same equilibrium. We 148 then calculate the Jacobian matrix around this equilibrium: 
$149 J=\left(\begin{array}{cc}r_{1}\left(1-\frac{2 N_{1}^{*}}{k_{1}}\right)-d_{1} & d_{2} \\ d_{1} & r_{2}\left(1-\frac{2 N_{2}^{*}}{k_{2}}\right)-d_{2}\end{array}\right)$

150 Based on this Jacobian matrix and the covariance matrix of population environmental responses,

151 we can solve the stationary covariance matrix (see Appendix 2).

152 The covariance matrices are then used to calculate temporal variability at the population

153 and metapopulation scales (see the next section). To evaluate the accuracy of our linearized

154 solutions, we also perform stochastic simulations based on the nonlinear equation (1). Simulation

155 results show that the linearization generally provides a good approximation unless environmental

156 stochasticity is large and exhibits a strong spatial asynchrony (see Figure A1). Note that in

157 natural ecosystems, environmental asynchrony is not expected to be strong, because the

158 environment generally exhibits positive spatial correlations (Moran 1953; Hudson \& Cattadori

159 1999). So the linearization approach may provide a good approximation in natural ecosystems.

Temporal variability at multiple scales

162 Within a metapopulation, variability can be measured at alpha, beta, and gamma scales, which

163 correspond to local population variability, spatial asynchrony among local populations, and

164 metapopulation variability, respectively (Wang and Loreau 2014). All these measures can be

165 derived from the mean $\left(N^{*}=\left(N_{1}{ }^{*}, N_{2}{ }^{*}, \ldots, N_{m}{ }^{*}\right)\right)$ and covariance matrix $\left(V_{N}=\operatorname{Cov}(\vec{X}(\infty))\right)$ of local

166 population sizes. We use the coefficient of variation $(\mathrm{CV})$, i.e. the ratio of the standard deviation

167 to the mean, to measure variability. For instance, the temporal $C V$ of population $i$ is: 
$168 C V_{i}=\frac{\sqrt{V_{N}(i, i)}}{N_{i}^{*}}$. Then, alpha variability is defined as the square of the weighted average of the 169 local population $C V$ :

$170 \quad \alpha_{C V}=\left(\sum_{i} \frac{N_{i}^{*}}{\sum_{j} N_{j}^{*}} \cdot C V_{i}\right)^{2}=\left(\frac{\sum_{i} \sqrt{V_{N}(i, i)}}{\sum_{j} N_{j}^{*}}\right)^{2}$

171 and gamma variability $\left(\gamma_{\mathrm{cv}}\right)$ is defined as the square of the temporal $C V$ of total metapopulation 172 size:

$173 \gamma_{C V}=\frac{\sum_{i, j} V_{N}(i, j)}{\left(\sum_{j} N_{j}^{*}\right)}$.

174 Beta variability or spatial asynchrony, is defined as the reciprocal of spatial population

175 synchrony: $\beta=1 / \varphi_{p}$, where the spatial synchrony is defined as:

$176 \varphi_{p}=\frac{\sum_{i, j} V_{N}(i, j)}{\left(\sum_{i} \sqrt{V_{N}(i, i)}\right)^{2}}$.

Metapopulation variability is then linked to local alpha variability and spatial (a) synchrony as

178 follows: $\gamma_{c v}=\alpha_{c v} \cdot \varphi_{p}=\alpha_{c v} / \beta$ (Wang and Loreau 2014). For the homogeneous case, we derive analytic formulae for this multi-scale variability, which is summarized in Table 1.

180

\section{Dispersal-induced stability and synchrony}

As demonstrated by previous studies, dispersal can simultaneously provide stabilizing and synchronizing effects on local population dynamics (Abbott 2011). The dispersal-induced stability $\left(D_{\alpha}\right)$ can be defined as the ratio of alpha variability without dispersal to that with dispersal (Abbott 2011): $D_{\alpha}=\alpha_{C V}^{d=0} / \alpha_{C V}$. Similarly, the dispersal-induced synchrony $\left(D_{\varphi}\right)$ can be defined as the ratio of spatial synchrony with dispersal to that without dispersal: 

$D_{\varphi}=\varphi_{p} / \varphi_{p}^{d=0}=\beta^{d=0} / \beta . D_{\alpha}$ and $D_{\varphi}$ quantify the local stabilizing and spatial synchronizing

188 effects of dispersal, respectively. The effect of dispersal on metapopulation stability $\left(D_{\gamma}\right)$ is

189 determined by the relative magnitudes of these two effects, i.e. $D_{\gamma}=\gamma_{C V}^{d=0} / \gamma_{C V}=D_{\alpha} / D_{\varphi}$. When

$190 D_{\gamma}$ is larger than 1 , the local stabilizing effect is larger than the spatial synchronizing effect, and

191 thus dispersal decreases metapopulation variability. Otherwise, dispersal increases

192 metapopulation variability.

\section{Results}

\section{Multi-scale variability in homogeneous metapopulations}

197

$$
D_{\alpha}=D_{\varphi}=\frac{\varphi_{p}}{\varphi_{e}}
$$

This implies that in homogeneous metapopulations, the effects of dispersal cancel out at the metapopulation level and thus dispersal has no net effects on gamma variability $\left(D_{\gamma}=1\right.$; see

207 Table $1 \&$ Fig. 1). These results, however, are based on linear approximations, which are 
208

209

210

211

212

213

214

215

216

217

218

219

220

221

222

223

224

225

226

227

228

229

230

appropriate when the environment fluctuates moderately. In a strongly fluctuating and asynchronous environment, simulations show that dispersal can provide weak stabilizing effects on gamma variability (Figure A1; see also Loreau et al. 2003).

The correlation of population environmental responses $(\rho)$ and the number of patches $(m)$ affect the multi-scale variability mainly through their effects on the spatial synchrony of population environmental responses $\left(\varphi_{e}\right.$; see Table 1). As $\rho$ increases and/or $m$ decreases (such that $\varphi_{e}$ increases), alpha and gamma variability both increase, and the beta variability decreases (Fig. 1). Besides, as the intrinsic growth rate $(r)$ increases, the temporal variability at alpha and gamma scales all decrease (Fig. 3). An increasing $r$ also weakens the spatial synchronizing effects of dispersal and environmental correlation and thereby increases spatial asynchrony. Note that dispersal is required for spatial parameters $(\rho$ and $m)$ to affect local alpha variability and for the local parameter $(r)$ to affect spatial asynchrony. When there is no dispersal $(d=0)$, alpha variability is independent of $\rho$ and $m$, and spatial asynchrony is independent of $r$ (Table 1).

\section{Effects of spatial heterogeneities on metapopulation variability}

In two-patch metapopulations, when keeping dispersal rates symmetric between the two patches, spatial heterogeneities in local parameters ( $r$ and $k$ ) generally increase gamma variability (Fig. 4). However, when the larger population (larger $k$ ) has faster local dynamics (larger $r$ ), such heterogeneity can contribute to reducing gamma variability if environmental responses are highly synchronous (Fig. 4f). When local populations have heterogeneous dynamics, increased (symmetric) dispersal rate tends to decrease gamma variability (Fig. 4a-d). However, when local populations differ in carrying capacity $(k)$, dispersal can be destabilizing, particularly when environmental responses are highly synchronous (Fig. 4e,f). Finally, note that in the cases with 
231 low or moderate heterogeneities in local parameters, symmetric dispersal has very limited effects

232 on metapopulation variability, just as it does in homogeneous metapopulations.

233 When keeping local dynamical parameters ( $r$ and $k$ ) homogeneous, asymmetry in

234 dispersal rates generally increases gamma variability (Fig. 5a,d). Under the extreme case where

235 one population does not disperse, the other population will have decreased population size and

236 increased variability as its dispersal rate increases (see Appendix 2). Consequently, the

237 metapopulation is dominated by one population (the non-dispersing one) and thereby exhibits

238 larger variability (Fig. 5a,d). However, spatial heterogeneities in local population dynamics can

239 alter this prediction qualitatively. For instance, if the non-dispersing population has faster local

240 dynamics (larger $r$ ), its dominance may contribute to reducing gamma variability, especially

241 when environmental responses are highly synchronous (Fig. 5e). Moreover, when local dynamics

242 are highly heterogeneous, the metapopulation is most stable when dispersal rates exhibit

243 moderate asymmetries. More specifically, gamma variability is lowest when the faster

244 population has a moderately higher dispersal rate (Fig. 5b,e), or when the larger population has a

245 moderately higher dispersal rate in asynchronous environments (Fig. 5c) or has a moderately

246 lower dispersal rate in synchronous environments (Fig. 5f).

\section{Discussion}

250 We have used dynamical models to study the role of dispersal, in interaction with other spatial 251 and local factors, in regulating the stability of metapopulations at multiple scales. Both the local 252 stabilizing and spatial synchronizing effects of dispersal have been documented in previous 253 studies (reviewed in Abbott 2011), and are again demonstrated by our models. One remarkable 
254 finding is that in homogeneous metapopulations, the local stabilizing effect of dispersal is always

255 identical to its spatial synchronizing effect; consequently, dispersal has no net effect on the

256 variability of the whole metapopulation (Fig. 1). This result is robust to moderate heterogeneities

257 in local and spatial parameters (Figs. $4 \& 5$ ), and is consistent with findings from experiments

258 with the same settings (i.e. experimental metapopulations with stable and homogeneous local

259 populations; see Vogwill et al. 2009). In deterministic metapopulation models, previous studies

260 have shown that random dispersal does not alter stability properties of the linearized system

261 when local population dynamics are homogeneous (reviewed in Briggs and Hoopes 2004). Here

262 we have further shown that dispersal does not affect the temporal stability of homogeneous

263 metapopulations in a fluctuating environment.

264 In heterogeneous metapopulations, spatial heterogeneities in local dynamical parameters

265 or dispersal rates generally increase metapopulation variability. However, when local dynamics

266 are heterogeneous, dispersal can provide stabilizing effects on metapopulation variability in

267 several ways. First, by linking populations with fast and slow dynamics, dispersal can decrease

268 gamma variability by either stabilizing both populations (Fig. A2) or providing stronger

269 stabilizing effects on the slower population and weaker destabilizing effects on the faster

270 populations (Fig. A3) (see also Briggs \& Hoopes 2004, Ruokolainen et al. 2011). In particular, a

271 moderately higher dispersal rate of the faster population can produce lowest gamma variability

272 (Fig. A5; see also Dey et al. 2014). Second, a much higher dispersal rate of the slower population

273 can leave the metapopulation dominated by the faster population, which decreases gamma

274 variability in highly synchronous environments (Fig. 5e). Third, in highly synchronous

275 environments, while symmetric dispersal rate between small and large patches can be

276 destabilizing (Fig. 4), a relatively higher dispersal rate of the smaller population can result in a 
277 zero net spatial flow of individuals, which decreases alpha and gamma variability (Fig. A5).

278 Finally, in highly asynchronous environments, dispersal can also provide stabilizing effects by

279 reducing spatial synchrony. Specifically, a higher dispersal rate of the larger population can

280 increase spatial evenness, which decreases spatial synchrony (Wang and Loreau 2014) and

281 thereby reduce gamma variability (Fig. A4). Similarly, when asymmetries in dispersal rates

282 operate in opposite ways - for instance the faster population has a much higher dispersal rate or a

283 moderately lower rate, or the larger population has a higher (smaller) dispersal rate in

284 (a)synchrounous environments - dispersal can increase the variability of the whole

285 metapopulation.

286 It is interesting to compare the role of dispersal in metapopulation stability with that of

287 interspecific competition in community stability. In metapopulations, populations interact

288 through dispersal in physical space; in competitive communities, populations interact through

289 interspecific competition in an abstract niche space. Remarkably, the effects of dispersal in our

290 models are just opposite to those of competition in community stability. As shown in previous

291 studies, competition can increase species variability but simultaneously decrease species

292 synchrony; in symmetric communities with identical species parameters, these two effects cancel

293 each other out and consequently competition has no effect on community stability (Hughes and

294 Roughgarden 1998, Ives et al. 1999, Loreau and de Mazancourt 2008, 2013). In asymmetric

295 communities, an increasing asymmetry in competitive abilities generally increases community

296 variability (Hughes and Roughgarden 1998, Loreau and de Mazancourt 2013); however, a

297 moderately higher competitive ability of the slower species, or a much higher competitive ability

298 of the faster species, can decrease community variability (Loreau and de Mazancourt 2013). All

299 these effects demonstrate the opposite roles played by interspecific competition in community 
300

301

302

303

304

305

306

307

308

309

310

311

312

313

314

315

316

317

318

319

320

321

322

stability and dispersal in metapopulation stability. These contrasting patterns could be

understood from the fact that competition reduces the population size of recipient species while dispersal increases the size of recipient populations. This is reflected in the Jacobian matrices in which competition and dispersal produce negative and positive interaction coefficients, respectively. In other words, dispersal acts as a form of spatial intraspecific mutualism in the dynamics of metapopulations.

Our results have important implications for landscape management. Corridors are commonly promoted as a conservation strategy to mitigate the effects of habitat fragmentation. Corridors have been demonstrated to promote dispersal and movement between habitat patches (Gilbert-Norton et al. 2010). But evidence is still lacking about the effects of corridors on population persistence (Haddad et al. 2011; but see Gonzalez et al. 1998), which is the ultimate reason for creating corridors. Our results suggest that corridors do not necessarily increase the stability and persistence of metapopulations (see also Earn 2000). In the specific case where local populations have nearly identical dynamical parameters, the synchronizing effect of dispersal cancels out their local stabilizing effect such that corridors have no net effect on the stability at metapopulation level. In reality, spatial heterogeneity can result in a stronger or weaker local stabilizing effect of dispersal compared to its synchronizing effect, and hence corridors may enhance or impair the stability of metapopulations. Our model suggests that in a heterogeneous landscape, the most efficient design of corridors is often one that generates asymmetric dispersal (Fig. 5). For instance, if local patches have different growth rates, the metapopulation is most stable when the faster-growing patch has a moderately higher dispersal rate than the slower-growing patch. Such asymmetric dispersal might be achieved by two mechanisms. First, when connected by corridors, the faster-growing population may have a 
323 higher level of dispersal activity spontaneously, e.g. by active dispersal (Pulliam 1988). Second,

324 it might be possible to design corridors that produce directional dispersal in some cases, for

325 instance by taking advantage of water and/or wind flow (Saumel and Kowarik 2010; Anderson et

326 al. 2012).

327

328 Concluding remarks

329 In this paper, we have explored how spatial processes govern the variability of metapopulations

330 at multiple scales in a stochastic environment. We show that within a metapopulation, dispersal

331 functions as a form of spatial intraspecific mutualism. While stabilizing local populations,

332 dispersal has very limited stabilizing effects on metapopulations if local population dynamics are

333 homogeneous. In highly heterogeneous metapopulations, however, dispersal can stabilize or

334 destabilize metapopulations through various mechanisms. Therefore, corridor designs, in order to

335 increase metapopulation stability and persistence, should be context dependent with explicit

336 consideration of spatial heterogeneities in population dynamics.

337 Our results are based on a simple metapopulation model and thus its limitations should be

338 kept in mind. First, in our models local populations always have stable equilibrium if there is no

339 environmental stochasticity. However, if local populations undergo complex dynamics (e.g. limit

340 cycles or chaotic attractors), dispersal can provide stabilizing effects through interacting with

341 nonlinearity and spatial heterogeneity (Briggs \& Hoopes 2004; Abrams \& Ruokolainen 2011;

342 Dey et al. 2014; see also Figure A1). Our model also ignores the effects of environmental

343 autocorrelation (Ruokolainen et al. 2009). We have shown that our first main result, i.e. the

344 stability of homogeneous metapopulations is not affected by dispersal, still holds for coloured

345 noise (see Appendix 4). Still, it would be worthwhile to investigate the interactive effects of 
346 coloured noise and spatial heterogeneities on the dispersal-stability relationship in future

347 research. Besides, our model ignores the effects of interspecific interactions, the inclusion of

348 which may alter some of our conclusions (Koelle \& Vandermeer 2005). For instance, corridors

349 can promote species co-occurrence and thus enhance competition or predation pressure, which

350 thereby may impair the persistence of the focal species (Loreau et al. 2003, Vogwill et al. 2009).

351 Finally, our model considers space implicitly, which could be extended to a spatially explicit one

352 to study the interactive effects between dispersal, spatial heterogeneity, and landscape

353 configuration (Holland \& Hastings 2008). Future studies should incorporate these complexities

354 to better understand the effects of dispersal on patchy populations in stochastic environments.

355

356

357

358

359

360

361

362

363

364

365

366

367

\section{Acknowledgements}

We thank Claire de Mazancourt for discussions, and Dries Bonte, Elad Shtilerman, and anonymous reviewers for helpful comments on earlier versions of the manuscript.

\section{References}

Abbott KC. 2011. A dispersal - induced paradox: synchrony and stability in stochastic metapopulations. Ecology letters 14:1158-1169.

Abrams PA, and Ruokolainen L. 2011. How does adaptive consumer movement affect population dynamics in consumer-resource metacommunities with homogeneous patches? Journal of Theoretical Biology 277:99-110. 
Anderson KE, Hilker FM, and Nisbet RM. 2012. Directional biases and resource - dependence in dispersal generate spatial patterning in a consumer - producer model. Ecology letters 15:209-217.

Blasius B, Huppert A, and Stone L. 1999. Complex dynamics and phase synchronization in spatially extended ecological systems. Nature 399:354-359.

Bowler DE, and Benton TG. 2005. Causes and consequences of animal dispersal strategies: relating individual behaviour to spatial dynamics. Biological reviews 80:205-225.

Briggs CJ, and Hoopes MF. 2004. Stabilizing effects in spatial parasitoid-host and predator-prey models: a review. Theoretical Population Biology 65:299-315.

Brown JH, Gillooly JF, Allen AP, Savage VM, and West GB. 2004. Toward a metabolic theory of ecology. Ecology 85:1771-1789.

De Roissart A, Wang S, and Bonte D. 2015. Spatial and spatiotemporal variation in metapopulation structure affects population dynamics in a passively dispersing arthropod. Journal of Animal Ecology. DOI: 10.1111/1365-2656.12400

Dey S, Goswami B, and Joshi A. 2014. Effects of symmetric and asymmetric dispersal on the dynamics of heterogeneous metapopulations: two-patch systems revisited. Journal of theoretical Biology 345:52-60.

Dey S, and Joshi A. 2006. Stability via asynchrony in Drosophila metapopulations with low migration rates. Science 312:434-436.

Earn DJ, Levin SA, and Rohani P. 2000. Coherence and conservation. Science 290:1360-1364.

Gilbert - Norton L, Wilson R, Stevens JR, and Beard KH. 2010. A Meta - Analytic Review of Corridor Effectiveness. Conservation Biology 24:660-668. 
390

391

392

393

394

395

396

397

398

399

400

401

402

403

404

405

406

407

408

409

410

411

Gonzalez A, Lawton J, Gilbert F, Blackburn T, and Evans-Freke I. 1998. Metapopulation dynamics, abundance, and distribution in a microecosystem. Science 281:2045-2047.

Gouhier TC, Guichard F, and Gonzalez A. 2010. Synchrony and stability of food webs in metacommunities. The American Naturalist 175:E16-E34.

Greenman J, and Benton T. 2005. The impact of environmental fluctuations on structured discrete time population models: resonance, synchrony and threshold behaviour. Theoretical Population Biology 68:217-235.

Haddad N, Hudgens B, Damschen E, Levey D, Orrock J, Tewksbury J, Weldon A, Liu J, Hill V, and Morzillo A. 2011. Assessing positive and negative ecological effects of corridors. In: Liu J, Hull V, Morzillo A, and Wiens JA, editors. Sources, sinks and sustainability. Cambridge: Cambridge University Press. p 475-503.

Heino M, Kaitala V, Ranta E, and Lindström J. 1997. Synchronous dynamics and rates of extinction in spatially structured populations. Proceedings of the Royal Society of London Series B: Biological Sciences 264:481-486.

Holland MD, and Hastings A. 2008. Strong effect of dispersal network structure on ecological dynamics. Nature 456, 792-795.

Hudson PJ, and Cattadori IM. 1999. The Moran effect: a cause of population synchrony. Trends in ecology \& evolution 14:1-2.

Hughes JB, and Roughgarden J. 1998. Aggregate community properties and the strength of species' interactions. Proceedings of the National Academy of Sciences 95:6837-6842. Inchausti P, and Halley J. 2003. On the relation between temporal variability and persistence time in animal populations. Journal of Animal Ecology 72:899-908. 
412 Ives A, Gross K, and Klug J. 1999. Stability and variability in competitive communities. Science $413 \quad 286: 542-544$.

414 Ives AR. 1995. Measuring resilience in stochastic systems. Ecological monographs 65:217-233.

415 Kendall BE, Bjørnstad ON, Bascompte J, Keitt TH, and Fagan WF. 2000. Dispersal,

416 environmental correlation, and spatial synchrony in population dynamics. The American $417 \quad$ Naturalist 155:628-636.

418 Koelle K, and Vandermeer J. 2005. Dispersal - induced desynchronization: from 419 metapopulations to metacommunities. Ecology letters 8:167-175.

420 Lande R, Engen S, and Sæther B-E. 1998. Extinction times in finite metapopulation models with $421 \quad$ stochastic local dynamics. Oikos:383-389.

422 Levine JM. 2003. A patch modeling approach to the community-level consequences of 423 directional dispersal. Ecology 84:1215-1224.

424 Liebhold A, Koenig WD, and Bjørnstad ON. 2004. Spatial synchrony in population dynamics. 425 Annual Review of Ecology, Evolution, and Systematics:467-490.

426 Loreau M, and de Mazancourt C. 2008. Species synchrony and its drivers: neutral and nonneutral 427 community dynamics in fluctuating environments. The American Naturalist 172:E48-E66. 428 Loreau M, and de Mazancourt C. 2013. Biodiversity and ecosystem stability: a synthesis of 429 underlying mechanisms. Ecology letters 16:106-115.

430 Loreau M, Mouquet N, and Gonzalez A. 2003. Biodiversity as spatial insurance in 431 heterogeneous landscapes. Proceedings of the National Academy of Sciences 100:12765$432 \quad 12770$.

433 Lundberg P, Ranta E, Ripa J, and Kaitala V. 2000. Population variability in space and time. $434 \quad$ Trends in ecology \& evolution 15:460-464. 
435 Moran P. (1953). The statistical analysis of the Canadian Lynx cycle. Australian Journal of $436 \quad$ Zoology, 1, 291-298.

437 Pimm SL. 1984. The complexity and stability of ecosystems. Nature 307:321-326.

438 Pulliam HR. 1988. Sources, sinks, and population regulation. American Naturalist 132:652-661.

439 Ripa J. 2000. Analysing the Moran effect and dispersal: their significance and interaction in $440 \quad$ synchronous population dynamics. Oikos 89:175-187.

441 Ripa J, and Ives AR. 2003. Food web dynamics in correlated and autocorrelated environments.

$442 \quad$ Theoretical Population Biology 64:369-384.

443 Ruokolainen L, Abrams PA, McCann KS, and Shuter BJ. 2011. The roles of spatial

444 heterogeneity and adaptive movement in stabilizing (or destabilizing) simple

445 metacommunities. Journal of theoretical Biology 291:76-87.

446 Ruokolainen L, Linden A, Kaitala V, and Fowler MS. 2009. Ecological and evolutionary

447 dynamics under coloured environmental variation. Trends in ecology \& evolution 24:555$448 \quad 563$.

449 Säumel I, and Kowarik I. 2010. Urban rivers as dispersal corridors for primarily wind-dispersed $450 \quad$ invasive tree species. Landscape and Urban Planning 94:244-249.

451 Schindler DE, Hilborn R, Chasco B, Boatright CP, Quinn TP, Rogers LA, and Webster MS.

452 2010. Population diversity and the portfolio effect in an exploited species. Nature 465:609$453 \quad 612$.

454 Steiner CF, Stockwell RD, Kalaimani V, and Aqel Z. 2013. Population synchrony and stability 455 in environmentally forced metacommunities. Oikos 122:1195-1206.

456 Strevens CM, and Bonsall MB. 2011. Density - dependent population dynamics and dispersal in 457 heterogeneous metapopulations. Journal of Animal Ecology 80:282-293. 
458 Van Kampen NG. 1992. Stochastic processes in physics and chemistry: Elsevier.

459 Vogwill T, Fenton A, and Brockhurst MA. 2009. Dispersal and natural enemies interact to drive $460 \quad$ spatial synchrony and decrease stability in patchy populations. Ecology letters 12:1194$461 \quad 1200$.

462 Wang S, and Loreau M. 2014. Ecosystem stability in space: a, b and g variability. Ecology letters $463 \quad 17: 891-901$.

464 
465

466

467

468

469

470

471

472

473

474

475

476

477

478

479

480

481

482

483

484

485

486

487

\section{Table 1 and Figures 1-5}

Table 1. Analytic solutions for multi-scale variability and spatial synchrony in homogeneous metapopulations. For clarity, we denote $d^{\prime}=m d /(m-1)$ and $\varphi_{e}=\frac{1+(m-1) \rho}{m}$. Note that by definition, we have $\beta=\alpha_{\mathrm{cv}} / \gamma_{\mathrm{cv}}$ and $\varphi_{p}=1 / \beta$.

Figure 1. Effects of the correlation in environmental responses $(\rho)$, number of patches $(m)$, and dispersal rate $(d)$ on multi-scale variability in homogeneous metapopulations. Parameters: $r=0.5$, $\sigma^{2}=0.05$, and $m=10$ for (a-c) and $\rho=0$ for (d-f).

Figure 2. Dispersal-induced stability $\left(D_{\alpha}\right)$ or synchrony $\left(D_{\varphi}\right)$ in homogeneous metapopulations. Note that $D_{\alpha}=D_{\varphi}$. Parameters for the bold line: $m=10, \rho=0, r=0.5$, and $\sigma^{2}=0.05$. Lines with marks have same parameters except: $m=5$ (triangle), $\rho=0.2$ (square), $r=1$ (circle).

Figure 3. Effects of the intrinsic population growth rate $(r)$ on multi-scale variability in homogeneous metapopulations. Black and red lines show results under $d=0$ and 0.2 , respectively. Solid and dashed lines show results under $\rho=0$ and 0.2 , respectively. Other parameters: $m=10, \sigma^{2}=0.05$.

Figure 4. Effect of spatial heterogeneities in local dynamical parameters and of (symmetric) dispersal rate on gamma variability in two-patch metapopulations. (a-c): gamma variability when environmental responses are perfectly asynchronous; (d-f) gamma variability when environmental responses are perfectly synchronous. The two patches differ in their intrinsic 
488 population growth rate $(r)$ and/or carrying capacity $(k)$, where a larger $s$ indicates a higher 489 heterogeneity. Other parameters: $\sigma^{2}=0.05$. See Figs. A2 \& A3 for the patterns of variability at 490 other scales.

491

492 Figure 5. Effects of asymmetric dispersal on gamma variability in two-patch metapopulations 493 (with homogeneous/heterogeneous local dynamics). (a-c): gamma variability when 494 environmental responses are perfectly asynchronous $\left(\varphi_{e}=0\right)$; (d-f) gamma variability when 495 environmental responses are perfectly synchronous $\left(\varphi_{e}=1\right)$. Symmetry in dispersal rates occurs 496 along the 1:1 diagonal; asymmetry increases as one moves away from this diagonal. Note that 497 along the 1:1 diagonal, gamma variability have similar patterns as those in respective panels in 498 Fig. 4. Other parameters: $\sigma^{2}=0.05$. See Figs. A4 \& A5 for the patterns of variability at other 499 scales. 


\section{Table $\mathbf{1}$ (on next page)}

Analytic solutions for multi-scale variability and spatial synchrony in homogeneous metapopulations 
1 Table 1. Analytic solutions for multi-scale variability and spatial synchrony in homogeneous

2 metapopulations. For clarity, we denote $d^{\prime}=m d /(m-1)$ and $\varphi_{e}=\frac{1+(m-1) \rho}{m}$. Note that by

3 definition, we have $\beta=\alpha_{\mathrm{cv}} / \gamma_{\mathrm{cv}}$ and $\varphi_{p}=1 / \beta$.

4

\begin{tabular}{lll}
\hline Variability or synchrony & Solution & Solution under $\mathrm{d}=0$ \\
\hline Population variability $\left(\alpha_{c v}\right)$ & $\alpha_{C V}=\frac{\left(r+d^{\prime} \cdot \varphi_{e}\right) \cdot \sigma^{2}}{2 r\left(r+d^{\prime}\right)}$ & $\alpha_{C V}^{d=0}=\frac{\sigma^{2}}{2 r}$ \\
Spatial asynchrony $(\beta)$ & $\beta_{1}=\frac{r / \varphi_{e}+d^{\prime}}{r+d^{\prime}}$ & $\beta_{1}^{d=0}=\frac{1}{\varphi_{e}}$ \\
Spatial synchrony $\left(\varphi_{p}\right)$ & $\varphi_{P}=\frac{r+d^{\prime}}{r / \varphi_{e}+d^{\prime}}$ & $\varphi_{P}^{d=0}=\varphi_{e}$ \\
Metapopulation variability $\left(\gamma_{c v}\right)$ & $\gamma_{C V}=\frac{\varphi_{e} \cdot \sigma^{2}}{2 r}$ & $\gamma_{C V}^{d=0}=\frac{\sigma^{2}}{2 r} \cdot \varphi_{e}$ \\
\hline
\end{tabular}

5 
Figure 1 (on next page)

Multi-scale variability in homogeneous metapopulations

Figure 1. Effects of the correlation in environmental responses $(\rho)$, number of patches $(m)$, and dispersal rate $(d)$ on multi-scale variability in homogeneous metapopulations. Parameters: $r=0.5, \sigma^{2}=0.05$, and $m=10$ for (a-c) and $\rho=0$ for (d-f). 

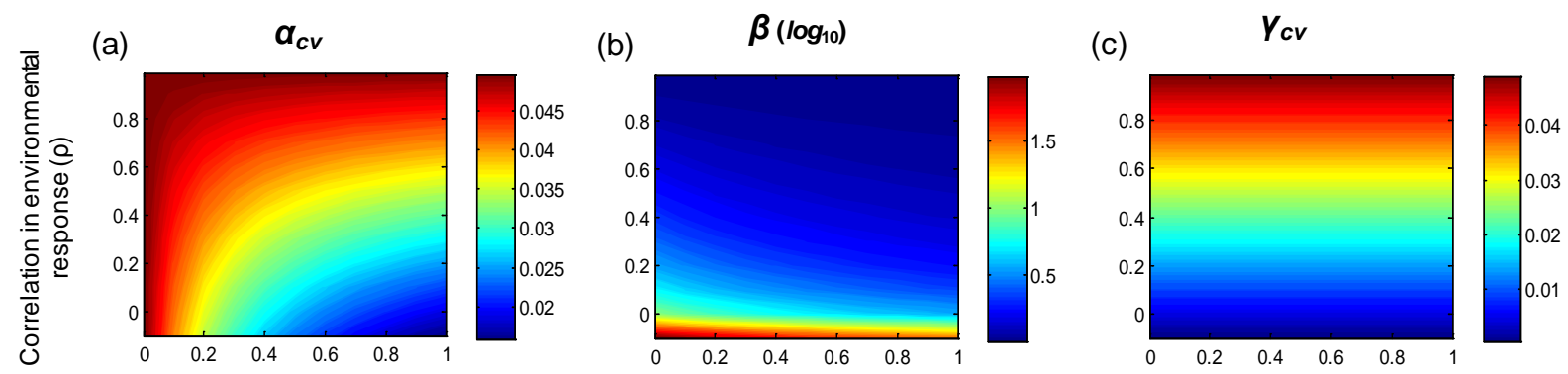

(d)

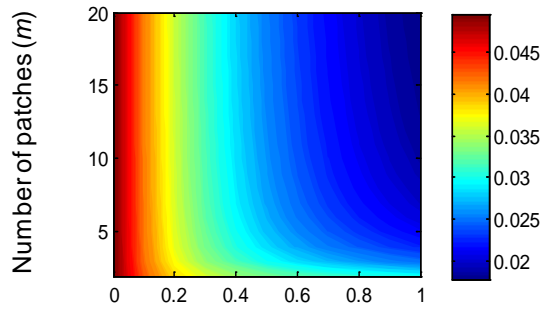

(e)

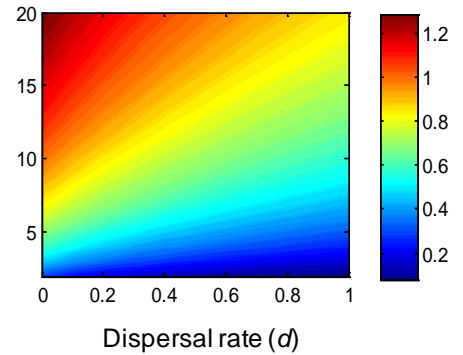

(f)

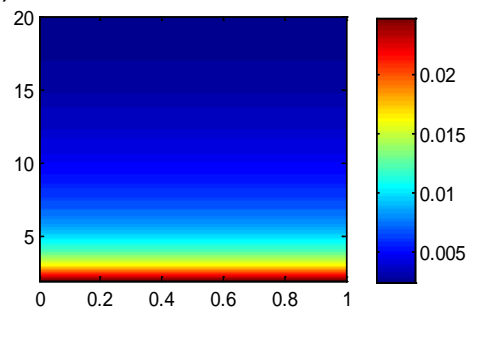


Figure 2 (on next page)

Dispersal-induced stability $\left(D_{\alpha}\right)$ or synchrony $\left(D_{\varphi}\right)$ in homogeneous metapopulations.

Figure 2. Dispersal-induced stability $\left(D_{\alpha}\right)$ or synchrony $\left(D_{\varphi}\right)$ in homogeneous metapopulations. Note that $D_{\alpha}=D_{\varphi}$. Parameters for the bold line: $m=10, \rho=0, r=0.5$, and $\sigma^{2}=0.05$. Lines with marks have same parameters except: $m=5$ (triangle), $\rho=0.2$ (square), $r=1$ (circle). 


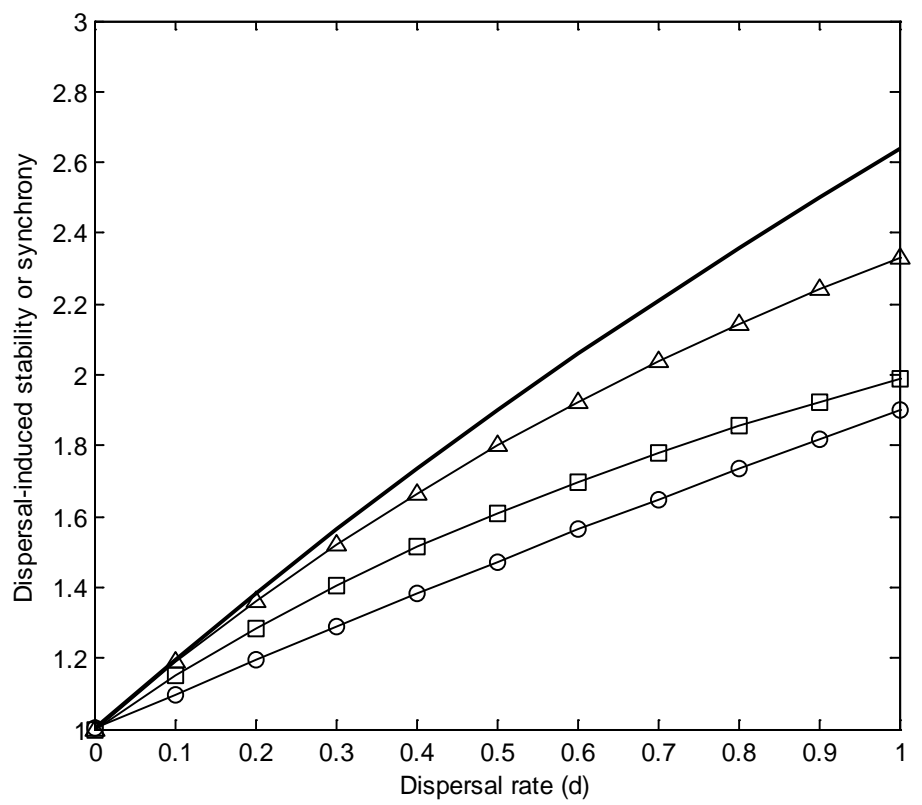


Figure 3 (on next page)

Effects of the intrinsic population growth rate $(r)$ on multi-scale variability in homogeneous metapopulations

Figure 3. Effects of the intrinsic population growth rate $(r)$ on multi-scale variability in homogeneous metapopulations. Black and red lines show results under $d=0$ and 0.2 , respectively. Solid and dashed lines show results under $\rho=0$ and 0.2 , respectively. Other parameters: $m=10, \sigma^{2}=0.05$. 
(a)

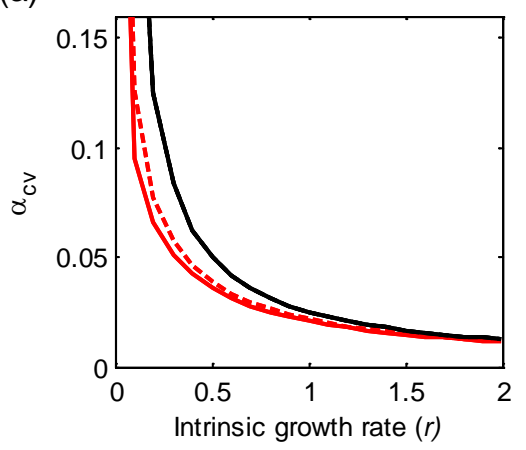

(b)

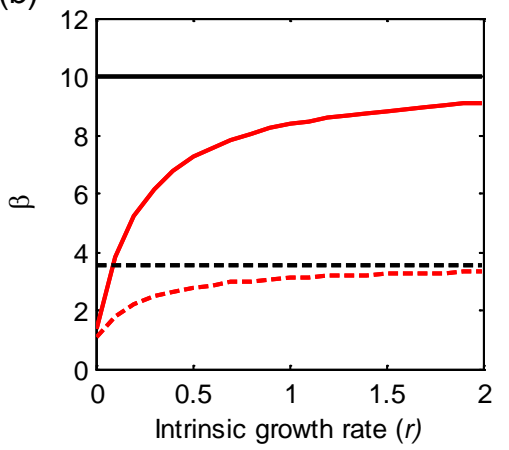

(c)

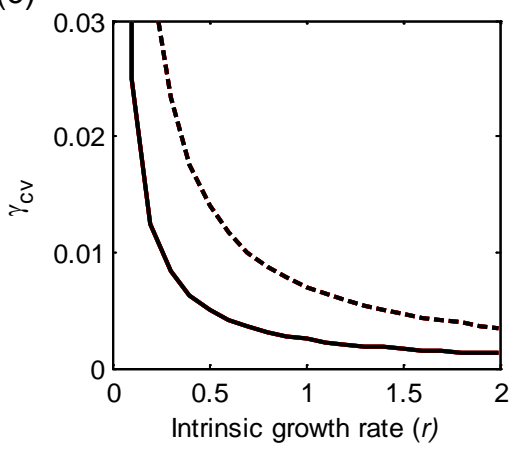




\section{Figure 4 (on next page)}

Effect of spatial heterogeneities in local dynamical parameters and of (symmetric) dispersal rate on gamma variability in two-patch metapopulations

Figure 4. Effect of spatial heterogeneities in local dynamical parameters and of (symmetric) dispersal rate on gamma variability in two-patch metapopulations. (a-c): gamma variability when environmental responses are perfectly asynchronous; (d-f) gamma variability when environmental responses are perfectly synchronous. The two patches differ in their intrinsic population growth rate $(r)$ and/or carrying capacity $(k)$, where a larger $s$ indicates a higher heterogeneity. Other parameters: $\sigma^{2}=0.05$. See Figs. $\mathrm{A} 2 \& \mathrm{~A} 3$ for the patterns of variability at other scales. 

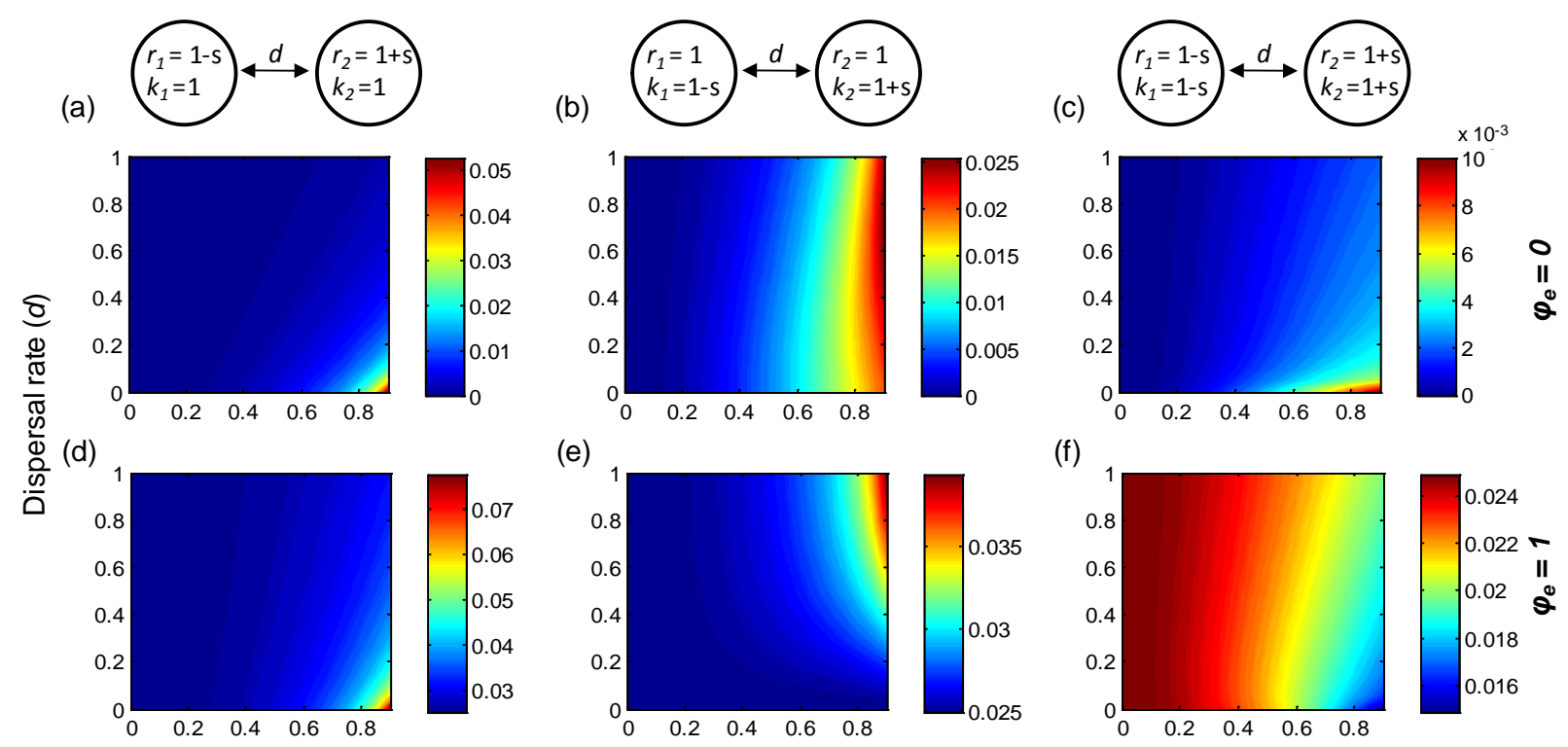

(e)

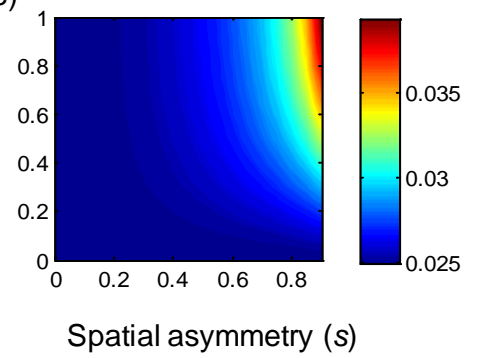

(f)

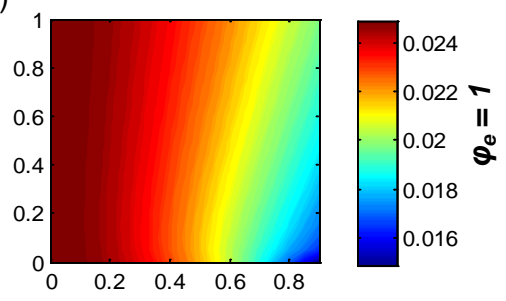




\section{Figure 5 (on next page)}

Effects of asymmetric dispersal on gamma variability in two-patch metapopulations

Figure 5. Effects of asymmetric dispersal on gamma variability in two-patch metapopulations (with homogeneous/heterogeneous local dynamics). (a-c): gamma variability when environmental responses are perfectly asynchronous $\left(\varphi_{e}=0\right) ;(d-f)$ gamma variability when environmental responses are perfectly synchronous $\left(\varphi_{e}=1\right)$. Symmetry in dispersal rates occurs along the 1:1 diagonal; asymmetry increases as one moves away from this diagonal. Note that along the 1:1 diagonal, gamma variability have similar patterns as those in respective panels in Fig. 4 . Other parameters: $\sigma^{2}=0.05$. See Figs. A4 \& A5 for the patterns of variability at other scales. 

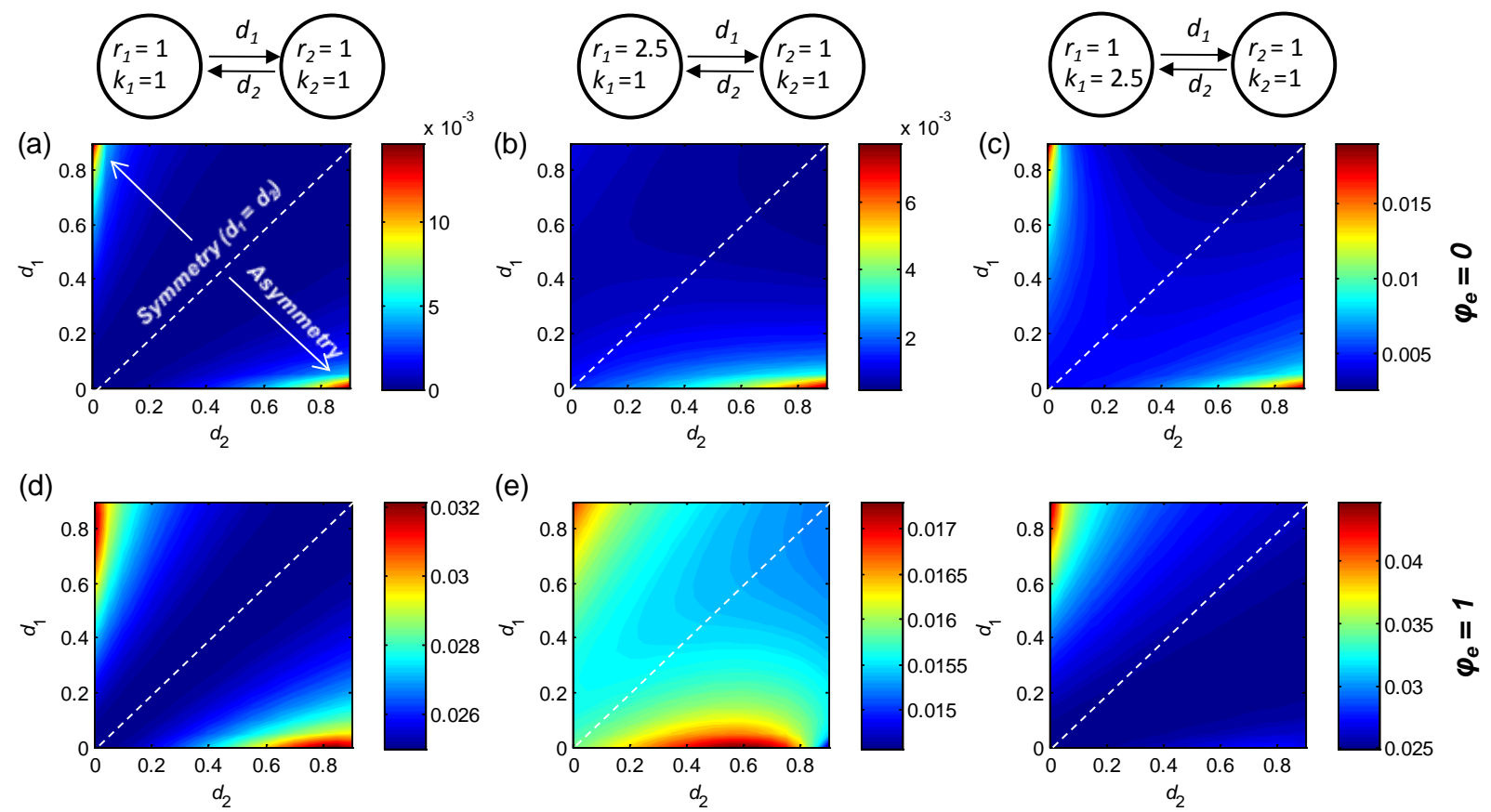

(e)
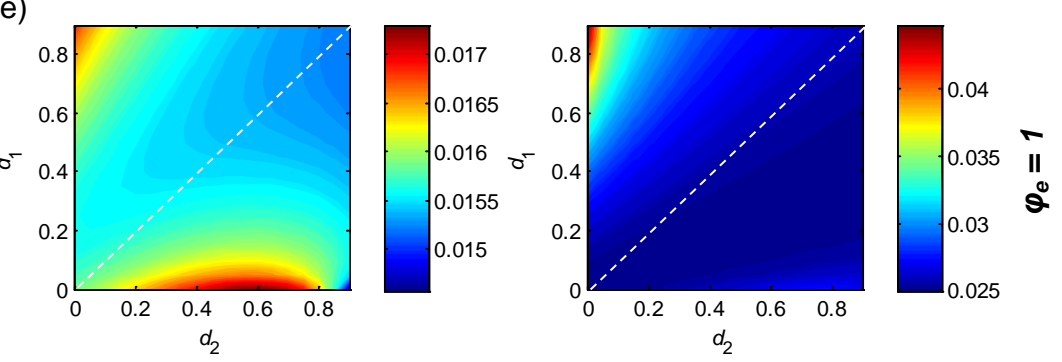